\title{
Alt werden im hohen Norden
}

Anna Sax

Finnland bereitet sich auf den demographischen Ernstfall vor: Die Alterung schreitet rasant voran, vor allem im dünnbesiedelten Norden. Bei einer Pressereise auf Einladung der Stadt Helsinki erhielt die SÄZ Einblick in Strategien für den Umgang mit der alternden Bevölkerung. Patentlösungen gibt es keine, aber die pragmatischen Finnen lassen sich einiges einfallen. Politische und technologische Innovationen sollen dazu beitragen, die Lebensqualität im Alter zu erhalten.

In den Jahren 1946 bis 1949 wurden jeweils mehr als 100 ooo Finninnen oder Finnen geboren - so viele wie nie zuvor und auch nicht danach. Seit 1970 hat sich die Zahl der Geburten bei ungefähr 60000 pro Jahr eingependelt. Die Babyboomer kommen nun ins höhere Alter, und besonders in den ländlichen Gebieten nimmt die Bevölkerungszahl seit einigen Jahren ab. Jüngere Leute zieht es in die Städte, ebenso wie die wenigen Immigrantinnen und Immigranten. Letztere kommen hauptsächlich aus dem sprachlich verwandten Estland und dem grossen Nachbarn Russland, aus Ländern also, die ihrerseits von demographischer Alterung betroffen sind. In Finnland wie in ganz Skandinavien gibt es riesige Gebiete, die sich langsam entvölkern, während die Städte weiter wachsen. 1,4 Millionen Menschen leben heute in der Grossregion Helsinki, rund ein Viertel der Landesbevölkerung. Die Behörden rechnen mit einer weiteren Bevölkerungszunahme in der Stadt und zugleich mit einer Entvölkerung auf dem Land.

\section{Stadt und Land}

Um Platz zu schaffen für mindestens 100 ooo weitere Einwohner baut Helsinki ganze neue Stadtteile. Neue Areale am Ostseeufer werden durch die Stadt und

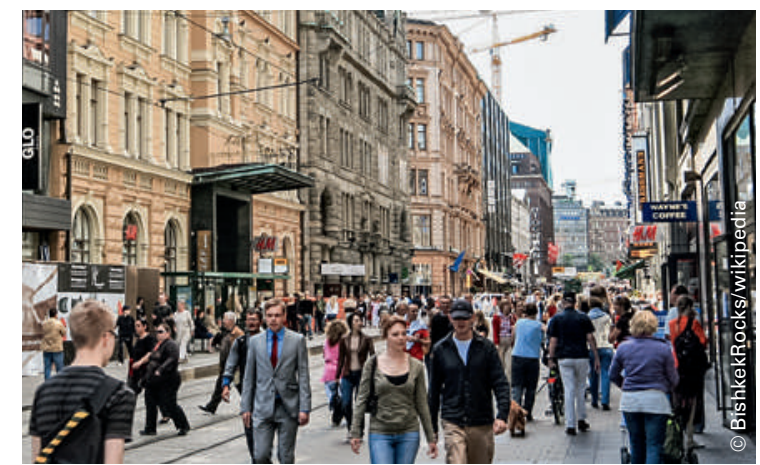

private Investoren erschlossen und überbaut, sogar der alte Stadtflughafen soll Wohnüberbauungen weichen. Bei jeder Überbauung sind Wohnungen, Arbeitsplätze und Erholungsgebiete gleichzeitig geplant. Unter dem Titel «Uutta Helsinkiä» - neue Horizonte für Helsinki - entstehen Stadtquartiere für alle Bedürfnisse: Ob naturverbunden oder urban, alleinstehend oder familiär, für alle soll passender Lebensraum entstehen. Eine schöne neue Welt ist auf der Webseite uuttahelsinkia.fi zu bewundern, aber wo bleiben da die alten Menschen? Tapio Kari, Leiter der städtischen Kommunikation, meint dazu: «Wir wollen eine soziale und altersmässige Durchmischung in allen Quartieren erreichen.» Die Möglichkeit dazu ist vorhanden: Der Boden ist praktisch vollständig in kommunalem Besitz, die Stadt kann direkten Einfluss nehmen auf die Überbauungen und die soziale Durchmischung der Bevölkerung.

Für die Betreuung und Pflege betagter Menschen gibt es in der Stadt und in den Vororten ein gut ausgebautes Angebot mit ambulanten und stationären Einrichtungen (vgl. SÄZ 35/2010, "Gesundheitsversorgung als öffentliche Aufgabe» [1]). Doch wie sieht es auf dem Land aus, wo die Nachbarn mehrere Kilometer entfernt wohnen und die Wege zum nächsten Gesundheitszentrum lang sind? Wie soll die Gesund-

Die jüngeren Finnen zieht es in die Städte. Bei der Versorgung der älteren Bevölkerung in den ländlichen Randregionen Finnlands gibt es zunehmend Engpässe. 
heitsversorgung im Alter sichergestellt werden, wenn die Jungen wegziehen und es an Fachpersonal mangelt? Die finnische Politik ist sich des Problems bewusst und hat 2013 ein Gesetz verabschiedet, das die Gemeinden dazu verpflichtet, die Situation der älteren Menschen auf ihrem Gebiet laufend zu überprüfen und Angebote zur Verfügung zu stellen, die notwendig sind, um ihre Gesundheit und ihr Wohlbefinden zu stärken. Altersbeauftragte sollen eingesetzt und Massnahmenpläne entwickelt werden in Zusammenarbeit mit Senioren-Beiräten. Den Gemeinden wird mit dem Gesetz auch die Verantwortung für die Langzeitpflege übertragen, wobei ambulante Angebote Vorrang haben vor Pflegeheimen. Da es in Finnland keine politische Zwischenebene gibt zwischen Staat und Gemeinden, kann die nationale Politik direkt auf die Gemeinden einwirken.

\section{Technologien als Unterstützung im Alltag}

Dass die auferlegte Aufgabe für die schrumpfenden Gemeinden nicht einfach ist, liegt auf der Hand. Auch deshalb sind vielerorts Initiativen entstanden, um technologische Lösungen zu entwickeln. Diese sollen das Leben der betagten Bürgerinnen und Bürger erleichtern und sie dabei unterstützen, ihre Selbstständigkeit länger zu bewahren. Bereits vorgestellt haben wir den «Menumat» (vgl. SÄZ 48/2014 [2]), der fertig zubereitete, tiefgefrorene Mahlzeiten zum einfachen Aufwärmen bereithält. Zwei finnische Altersorganisationen starteten 2010 mit finanzieller Unterstützung der nationalen Lottogesellschaft das Projekt «KÄKÄTE» mit dem Ziel, Technologien für ältere Menschen zugänglich zu machen und der Industrie das Zielpublikum der Senioren näher zu bringen. Mit Umfragen ermittelten Sie, wie Rentnerinnen und Rentner Informationstechnologien nutzen und welche Verbesserungen sie von der Industrie erwarten. Herausgekommen sind schon eine ganze Reihe einfach zu bedienender Produkte für den Alltag, etwa multifunktionale Smartphones, eine MedikamentenErinnerungshilfe oder ein Nachtlicht mit Bewegungsmelder. Viele Technologiefirmen haben inzwischen das Potential der betagten Kundschaft erfasst und lassen ihre Geräte von Seniorinnen testen, bevor sie sie auf den Markt bringen. Auch private Dienstleistungsunternehmen haben die älteren Leute als Kunden entdeckt. Marika Nordlund, Projektleiterin von «KÄKÄTE», freut sich über das zunehmende Interesse der Wirtschaft. Sie will auch festgestellt haben, dass sich bei der finnischen Bevölkerung eine Mentalitätsänderung anbahnt: «Die Leute vertrauen immer weniger darauf, dass sich die Gemeinden umfassend um sie kümmern werden. Sie sind zunehmend bereit, Dienstleistungen einzukaufen, die sie benötigen.»

\section{Ältere sorgen für Ältere}

In Finnland wie in den anderen europäischen Ländern bedeutet die demographische Alterung eine gewaltige Herausforderung nicht nur für die Gesundheitsversorgung, sondern auch für das Weiterbestehen der Randregionen als Lebens- und Wirtschaftsräume. Das Stadt-Land-Gefälle ist in diesem nordischen Land mit äusserst geringer Immigration besonders ausgeprägt. Ausgeklügelte Technologien werden vielleicht das Leben von alten Menschen in dünn besiedelten Regionen etwas erleichtern, doch auch die erfinderischen Finnen sind sich bewusst, dass zusätzlich gesellschaftspolitische Veränderungen notwendig sind. Wie es bei der Stadtentwicklung von Helsinki bereits der Fall ist, wird sich der Fokus auch auf dem Land vermehrt auf das Zusammenleben und die gegenseitige Unterstützung richten. Das neu geschaffene Gesetz zur Stärkung der älteren Bevölkerung schreibt zwar das Recht auf eine angemessene Pflege und Gesundheitsversorgung fest, setzt aber in erster Linie bei der Selbstorganisation und Mitbe-

\section{Den Gemeinden wird mit dem Gesetz} auch die Verantwortung für die Langzeitpflege übertragen.

stimmung auf Gemeindeebene an. So werden wahrscheinlich auf dem Land die älteren Menschen vermehrt gegenseitig zueinander schauen müssen, da Töchter, Söhne und medizinisches Fachpersonal fehlen. Da ist auch das Recht auf Urlaub für die Betreuung von kranken oder behinderten Angehörigen, wie es Finnland kennt, nur ein kleines Puzzleteil in einem komplexen Zukunftsszenario.

Aus Schweizer Sicht ist Finnland so etwas wie ein Fenster in die Zukunft: Der Babyboom setzte dort etwa zehn Jahre früher ein als hierzulande, entsprechend stärker sind die Randregionen bereits heute von Engpässen in der Versorgung der älteren Bevölkerung betroffen. Wir können davon lernen, wie es dieses Land schaffen wird, unter besonders schwierigen Voraussetzungen den demographischen Wandel heil zu überstehen.

\section{Referenzen}

1 Sax A. Gesundheitsversorgung als öffentliche Aufgabe. Schweiz Ärztezeitung. 2010;91(35):1349-51.

2 Sax A. Menumat. Schweiz Ärztezeitung. 2014;95(48):1836 